\title{
New Insights on Time and Quantum Gravity
}

\author{
Ozer Oztekin \\ Pamukkale University Faculty of Medicine, \\ Department of Obstetrics and Gynecology, Denizli, TURKEY
}

\begin{abstract}
According to Einstein, a universal time does not exist. But what if time is different than what we think of it? Cosmic Microvawe Background Radiation was accepted as a reference for a universal clock and a new time concept has been constructed. According to this new concept, time was tackled as two-dimensional having both a wavelength and a frequency. What our clocks measure is actually a derivation of the frequency of time. A relativistic time dilation actually corresponds to an increase in the wavelength of time. At the point where time wavelength and time frequency is equal, where light is positioned, quantum-world and macro-world are seperated. Gravity was redefined with respect to time and the new two dimensional time fabric of the universe was speculated to be the source of dark energy causing the universe to expand. According to this new point of view quantum realm and macro-world can be better understood. This new time concept provide a basis for our understanding of quantum gravity and provide the long-sought answers to well known problems of it. A prediction of the presented theory is that the universe will expand at various rates at different regions due to the fact that particular surroundings will create different gravities and cause a different gravity- time wavelength effect yielding various time delays for calculating this rate of expansion.
\end{abstract}

Keywords: General Relativity, Gravitation, Time and frequency, Quantum mechanics

DOI: $10.7176 /$ APTA/83-08

Publication date: May $30^{\text {th }} 2020$

\section{INTRODUCTION}

What is time? Does time have a flow rate? What does the speed of time flow mean ? How fast does it flow? What determines the speed of time flow? What happens to time when we approach to the limits of universe at macro and micro levels? Is there a universal time? How is time and duration related ? How is time and mass related? Why the time has one-way direction? Is time preserved within the universe? Is it time to change our understanding of time conept? As we can see, questions are many. Do the answers to these questions have the potential to provide the long-sought answers to the problems of quantum gravity or the theory of everything?

\section{CREATING A NEW TIME CONCEPT}

Let's look deeper into our "time concept" to find answers to above questions. Consider an object with rest mass of $m$ starts moving with a velocity $v$ with respect to an observer who is stationary. Suppose that $v$ is a significant fraction of the speed of light. Einstein's theory of special relativity predicts that time slows down or speeds up depending on how fast you move relative to something else and being affected by acceleration it does not flow at a steady rate $[1,2]$. In Einstein's theory of special relativity, time dilation describes a difference of elapsed time which stems from the difference between the rates of time flow in different conditions. Apparently, what is meant by "rate of time flow" here is the speed of the "ticks"' of the clock in each condition. Nevertheless, the object experiences a time dilation and will have a relativistic mass of $m^{\prime}$. Let's designate the elapsed time as $t$ for the observer and $t^{\prime}$ for the object.

\subsection{Relativistic Time-Mass Formula}

According to Einstein, a universal time does not exist [3]. But what if time is different than what we think of it? We will discuss this below but for the moment let's suppose that there is a universal time and for simplicity let's choose the cosmic background microwave radiation as a frame of reference for this universal clock. According to this universal clock, the amount of time (not the duration !) for each condition (for observer and the object moving) should be the same. How can this be possible? This could only be possible if the time is condensed for the object moving. So, we should describe a new concept at this point: time density. Time density can be described as the amount of time quanta (Planck time) within each time unit. Let's denote time density as "' $z$ " for the moment. We can conclude that time density is inversely proportional to the elapsed time. Hence we can say that 
for the universal clock the amount of time for the observer $(\mathrm{t} x \mathrm{z})$ is equal to the amount of time for the moving object $\left(t^{\prime} x z^{\prime}\right)$, (see Fig 1 below). We can write the equation as :

$\mathrm{t} \times \mathrm{z}=t^{\prime} \times z$

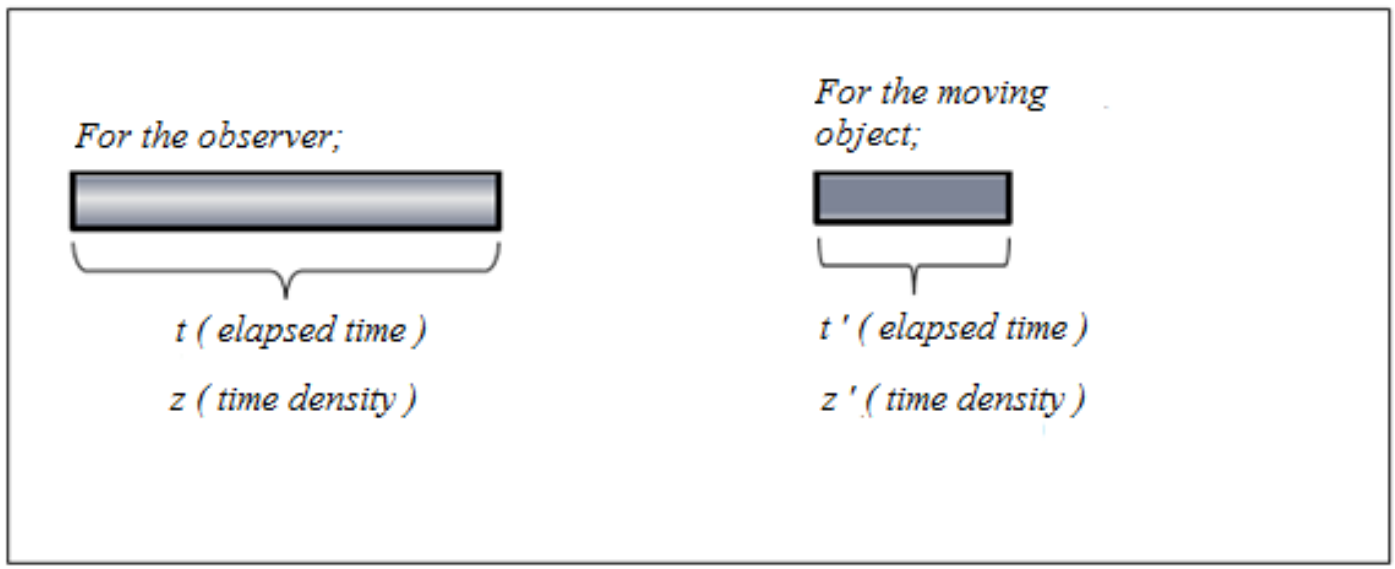

Figure 1. Schematic representation for the elapsed time ( $t$ for the observer, $t^{\prime}$ for the moving object ) and time density ( $\mathrm{z}$ for the observer, $z^{\prime}$ for the moving object). Note that for the moving object, time slows down (dilated) and time density increases meaning time is condensed. Elapsed times are represented by the length of the bars and time densities are represented by the gradient difference of the bars $\left(z^{\prime}>z\right)$.

Relativistic formulas for dilated time and relativistic mass for this object are as follows:

$t^{\prime}=t \cdot \sqrt{1-\frac{v^{2}}{c^{2}}}$

where $t$ ' is dilated time which is the elapsed time experienced by the object, $t$ is the elapsed time experienced by the observer, $v$ is velocity of the object and $c$ is the speed of light [4].

$$
m^{\prime}=\frac{m}{\sqrt{1-\frac{v^{2}}{c^{2}}}}
$$

where $m$ ' is relativistic mass of the object, $m$ is rest mass of the object, $v$ is the velocity of the object and $c$ is the speed of light [5].

From these equations it can be derived that $: \frac{t}{t \prime}=\frac{m^{\prime}}{m}$. Combining with the above equation $\left(\mathrm{t} \times \mathrm{z}=t^{\prime} x z^{\prime}\right)$ we can conclude that $\frac{m}{m^{\prime}}=\frac{z}{z^{\prime}}$ or $m x z^{\prime}=m^{\prime} x z$. This result relates the time with mass and implies that every object with a mass has a time density and this time density is directly proportional to the amount of mass the object has.

\subsection{Time Wavelength and Time Frequency}

We know that when a mass is subjected to relativistic speeds, the time slows down and when this speed reaches to the speed of light, the time stops. So, it is clear that there is a close relationship with the velocity of time flow and the light speed. The speed of light is the limit for the time to flow. They both have speeds and they both have limits to their speeds. Another common property is that they both have different speeds in different circumstances. Continuing this analogy brings us to the thought that time may be a kind of wave.To understand this concept better, let's turn back to above example. From the figure below we can conclude that what we previously called as time density ( $\mathrm{z}$ ) actually turns out to be the time wavelength and ticks of the clock represent a measure of time frequency (Fig. 2). To measure the elapsed time we measured the time frequency. So time in its historical meaning that is used until today turns out to be equivalent to the time frequency $\left(t_{f}\right)$ of our new time concept. In the 
figure, distance between the dots represents the time wavelength $\left(t_{\lambda}\right)$ and the frequency of the dots represents the time frequency $\left(t_{f}\right)$.

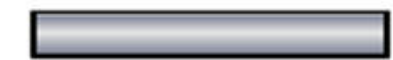

Elapsed time $(\mathrm{t})$ for $\mathrm{m}$

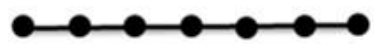

Ticks of clock for m. Each black circle represents a tick of the clock.

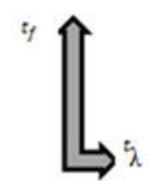

Representation of the magnitudes of the frequency ( $t f$, vertical axis ) and the wavelength ( $t \lambda$, horizontal axis ) of the time for $m$.
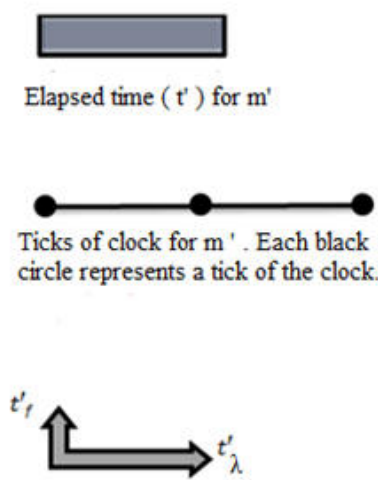

Representation of the magnitudes of the frequency ( $t^{\prime} f$. vertical axis) and the wavelength ( $t^{\prime} \lambda$, horizontal axis ) of the time for $\mathrm{m}^{\prime}$.

Figure 2. Schematic representation of time wavelength $\left(t_{\lambda}\right)$ and time frequency $\left(t_{f}\right)$ in relation to elapsed time and ticks of clock in relativistic speeds. Note that both $t_{\lambda}$ and $t_{f}$ have units of $s^{-1}$.

Now we face with a new time concept having two parameters as frequency and wavelength. What our clocks measure is actually a derivation of the frequency of time. A relativistic time dilation actually corresponds to an increase in the wavelength of time.

Let's consider our previous equation: $t x z=t^{\prime} x z^{\prime}$. We just concluded that ' $z$ ', is time wavelength $\left(t_{\lambda}\right)$ and ' $t$ '” is time frequency $\left(t_{f}\right)$. So correcting the equation according to our new time concept we find that:

$t_{\lambda} \cdot t_{f}=t_{\lambda \cdot t_{f}^{\prime}}^{\prime}$

It can be deduced logically that $t_{\lambda} \cdot t_{f}$ is constant throughout the universe. Graphic below depicts the $t_{\lambda}$ and $t_{f}$ relevancy of our universe (Fig. 3).

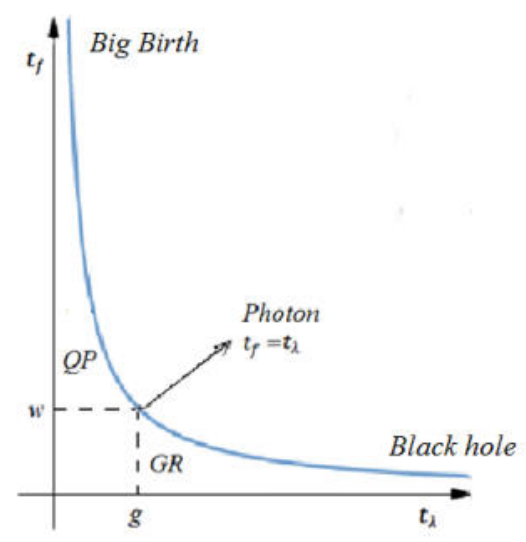

Figure 3. Time wavelength $\left(t_{\lambda}\right)$ and time frequency $\left(t_{f}\right)$ graphic depicting $t_{\lambda} x t_{f}$ is constant. Note that at extremely high $t_{\lambda}$ values, black hole conditions apply while at extremely high $t_{f}$ values Big Birth (see below) conditions apply. 
Our mass-time density equation $\left(m x z^{\prime}=m^{\prime} x z\right)$ is now as follow:

$\mathrm{m} \cdot t_{\lambda}^{\prime}=m^{\prime} \cdot t_{\lambda}$

\section{GRAVITY WITH RESPECT TO TIME}

At this point, I postulate a new definition for gravity: ' Gravity is the resistance applied by time on a mass against its' movement or acceleration'.' In an analogy to Newton's second law of motion [6], this definition states that gravity of an object is directly proportional to the magnitude of its mass and time wavelength it is bound to. Hence, we get a new proportionality; $\mathrm{g} \propto \mathrm{m} . t_{\lambda}$

When the Fig. 3 is explored we can see that as we go towards the right side on $\mathrm{x}$ axis, both gravity and $t_{\lambda}$ increases and reaches their maximum (infinity ?). At the point where $t_{\lambda}$ and $t_{f}$ is equal, quantum-world and macro-world are seperated. When we go to the right side beyond the point " $\mathrm{g}$ " on $\mathrm{x}$-axis, gravity emerges and the rules of General Relativity (GR) applies. When we go upwards on y-axis beyond the point "w', wave function emerges and the rules of Quantum Physics applies. Thus, these two worlds are sperated within a continuum of time. Changing the parameters of time changes our worlds. In the intersection between them where $t_{\lambda}$ and $t_{f}$ is equal the light is placed which is massless and has both particle and wave properties meaning it has the properties of both worlds. Going towards the right end of x-axis brings us to zero or starting point on y-axis. Is this the end of everything? Does this bring us to a singularity? To understand the situation for time better let's draw a 3-dimensional time graphic (Fig. 4). Notice that both $t_{\lambda}$ and $t_{f}$ have their maximum and minimum limits. On this graphic we see that when maximum $t_{\lambda}$ is reached we get to the starting point again. At this moment $t_{f}$ is zero which means we are at the starting point again. From another point of view, we are at maximum $t_{f}$ and minimum $t_{\lambda}$ which also means that we are at the start of a new cycle if we go further !

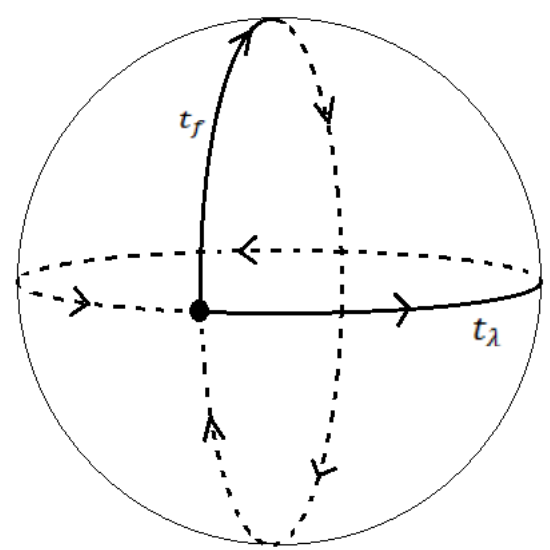

Figure 4. Three-dimensional representation of time wavelength $\left(t_{\lambda}\right)$ and time frequency $\left(t_{f}\right)$ relevance. Note that as $t_{\lambda}$ (represented on x-axis) increases $t_{f}$ (represented on y-axis) decreases and vice versa. At maximum value of $t_{\lambda}, t_{f}$ is minimum and both are at the starting point represented by a black point.

For the embodiment of this new time concept let's think that we symbolise the time as an hourglass. One compartment of the hourglass represents the frequency of time $\left(t_{f}\right)$ and the other compartment represents the time wavelength $\left(t_{\lambda}\right)$. Let's assume that as we go along x-axis, the hourglass turns clockwise (Fig. 5). 


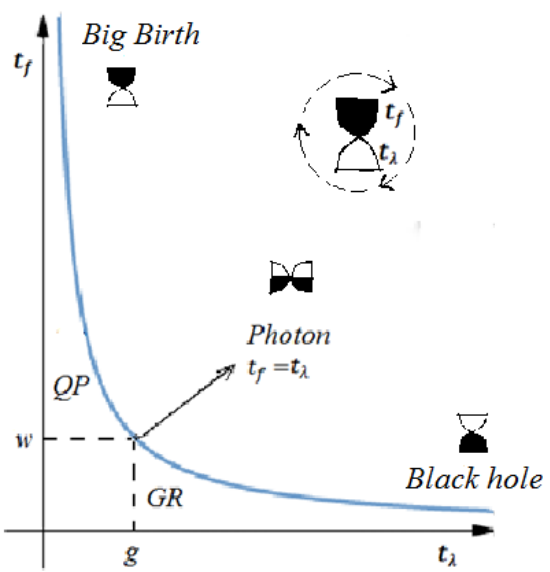

Figure 5. Representation of time as an hourglass model. Black compartment of the hourglass represents $t_{\lambda}$ and white compartment represents $t_{f}$. At photon level both are in equilibrium.

In order to understand the concept better it might be helpful to make an anology at this point. Let's think of a sea. There are continuous waves on it propogating at a certain interval. Suppose a boat (boat A) was anchored in the sea. Waves are coming towards the Boat A and going backwards. A device (device A) on the boat counts the waves that hit the boat. Let's assume device A counts 10 waves and plotted each hit on a paper which is printed out. Now let's go further and further in the sea until we come to an ocean. Here again there is a boat (boat B) and another device (device B) counting the waves. But this time the ocean is big and the waves are bigger. So interwal between the waves are longer. Assume that device B counts 10 waves also and plotted these dots on a paper, too. Now let's go very far away to see that there is a point where all the waves are originating from a single point (point $\mathrm{X}$ ) where there is a device $\mathrm{C}$. Waves closer to this point have very big intervals between them. Every single wave originating here hits both the boats A and B while propogating. In this example the water is the space, the waves which have both wavlengths and frequencies are the time and the devices counting the hits are the clocks. The seaside is the quantum world. The same amount of water waves (time quantas) given to each boats and devices A and B counted the same waves, actually. The drawing made by device A is this: . . . . . . (10 dots). The drawing made by device B is this: . $\quad . \quad$. $\quad . \quad$. . . . . . . . . (10 dots) which is longer. The paper used by device $\mathrm{C}$ is the longest of all. This means that the durations are different for every device. Newton took the drawing of device A into the account. Einstein considered drawings of both device A and device B. Device C represents the clock of CMBR. Nevertheless the time propogates as a wave but we perceive it as an arrow. 


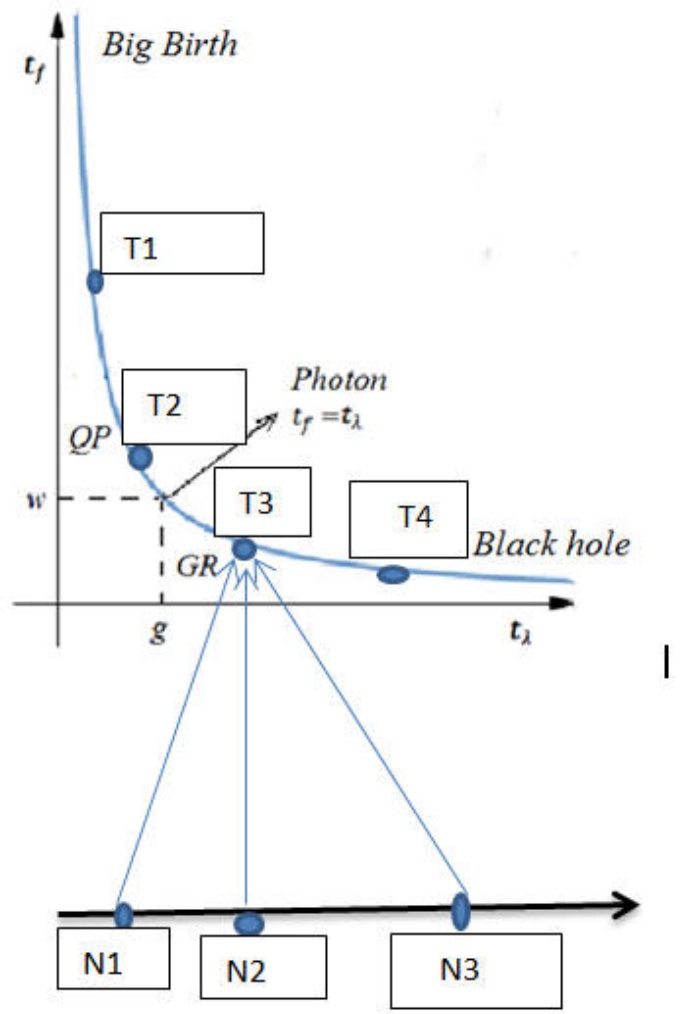

\section{Newtonian Time}

Figure 6. Comparison of Newtonian time and our time concept. N1,N2 and N3 represents the order of events on a time arrow. T1, T2, T3 and T4 are the time coordinates determining the pace at which time passes at a point in space.

Newtonian time is absolute. An event taking place at the time moment N3 takes place after N1 and N2 (fig. 6). Event occuring at time moment N2 takes place after N2 and before N3. But according to our new concept, events in N1, N2 and N3 all has the same time coordinates of T3 which has a constant wavelength and frequency meaning the same pace of time was applied on both events. Time passes forward for both N1,N2,N3 and T3. The coordinate graphic here is not meant to show the time arrow. We can not see the time arrow on $\mathrm{x}$, y coordinates. What a Newtonian clock measures as time duration is actually a measure of time frquency (total amount of time quantas) used for that event. Time wavelengths for both events are fixed and the same. So, Newtonian time does not take $\mathrm{x}$ coordinate ( time wavelength) into account and makes measurement on y coordinate ( time frequency) only. 


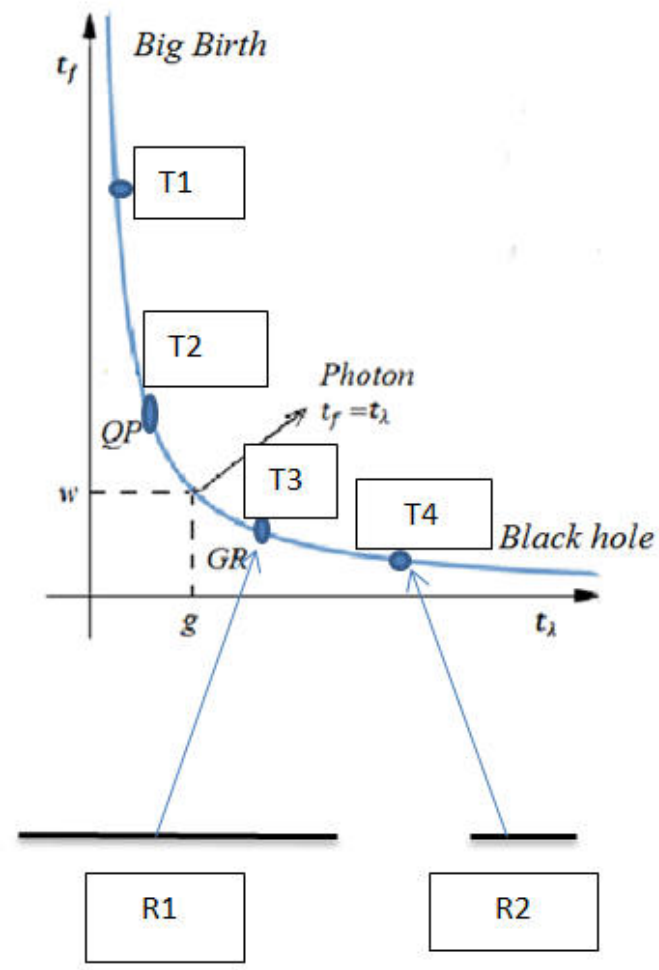

\section{Einsteinian - Relative time / time dilation}

Figure 7. Explanation of Einsteinian / Relative time concept on our time coordinate graphic. T1, T2, T3 and T4 are the time coordinates determining the pace at which time passes at a point in space. R1 and R2 represents events occuring at two distinct points when objects are either moving relatively to eachother, or differently, depending on their proximity to a gravitational mass.

Einstein belevied that there was no absolute time. A universal time does not exist and hence there is a relativity of simultaneity according to him. On the arrow of time he once said in a letter to Michele Besso that " Our subjective experience of time has no objective significance, and the Big Bang must have happened independently of the time's arrow ", (7). Nevertheless, according to him the elapsed time in events R1 and R2 ( above graphic) are different if the objects are either moving relatively to eachother, or differently, depending on their proximity to a gravitational mass. In the graphic above elapsed time in R2 is smaller than that of R1. These events has time coordinates T3 for R1 and T4 for R2 on the graphic. In the graphic R2 is near a greater gravity or has a relativistic speed. According to Einsteinian time concept, the elapsed time is smaller for R2 than that of R1 due to a time dilation, which is due to an increase in the time wavelength of coordinate of T4 according to the new time concept presented in this manuscript. Time is dilated due to an increased wavelwength of time. Elapsed time is smaller for R2 due to the decreased frequency of T4 time coordinate. If we were to accept Cosmic Microwave Background Radiation as a reference frame we may think of a Universal time, without conflicting with Special or General Relativity. Time coordinate graphic used in this paper is like a graphic showing the time coordinates within our Universe at a given moment. Everything, every particle, every object has a time coordinate which can be shown in this graphic. Coming back to events in R1 and R2, we can conclude according to this new time concept that taking into account both time wavelength and frequency, total amount of time quantas spent in R1 and R2 is equal to eachother for a Universal clock. We know that Einstein argued and questioned the events occuring at T1 and $\mathrm{T} 2$. He worked on time dilation (wavelength of time according to the new concept)) hence worked on the $\mathrm{x}$ coordinate (time wavlength) of the graphic but reluctant to work on the left side of this time graphic. Thus, he was not able to see the whole picture.

\section{BIG BANG OR BIG BIRTH ?}

At maximum $t_{\lambda}$ and minumum $t_{f}$, the present author further proposes that increasing gravity is not possible at this stage and trying to increase the gravity will cause the time to shift (turns the hourglass upside down) and birth 
of a new universe (or galaxy at lower scales) starts. As it can be clearly understandable from fig. 4 and fig. 5 this time shift is not a time reversal or change in the direction of the time arrow. In fact, it is the direct result of the continuing in the direction of the time arrow. What would this time shift mean? It means that a new cycle starts and we get to the beginning of the time graphic in Fig.3. Here at the beginning as we pass from the world of GR to a new world of QP, a massless "' quark gluon plasma (QGP) ", [8] emerges at the site of the black hole.

Here, it appears that just like the light, both $t_{\lambda}$ and $t_{f}$ have their own maximum limits which can't be exceeded (fig. 4). At this point let's remember Cherenkov radiation [9] in which an electromagnetic radiation is emitted when a charged particle passes through a medium at a speed greater than the velocity of light in that medium. Similar to this situation, present author suppose that when a mass with maximum gravity is forced through the maximum $t_{\lambda}$ and minimum $t_{f}$, a QGP with maxiumum $t_{f}$ and minimum $t_{\lambda}$ gradually appears along with a radiation. Here, the transition is smooth and instantaneous and no singularity appears. It is just like an hourglass again; things are passing smoothly from one compartment to the other, instantaneously. I term this transition as 'Big Birth', which is in opposition to Big Bang but correlates well with Big Bounce Theory. Nevertheless, we have matter-gravity, maximum $t_{\lambda}$ and Hawking Radiation [10] on the left side of the equation and QGP-wave function, maximum $t_{f}$ and Cosmic Microwave Background Radiation (CMBR) on the other side. This CMBR is a conjugate of $t_{\lambda}$ and $t_{f}$ at the time of Big Birth and can serve as a universal clock for the time evolution in our universe.

\section{SOLUTIONS TO FAMOUS PROBLEMS}

If we were able to observe the event horizon of a black hole we would see that time stopped eventually and everything was frozen. We would think ourselves as watching the past since the time velocity of the black hole is slower then ours. We would feel that we were ahead of the black hole as far as the time was considered. But for the black hole itself, behind the event horizon, the instantaneous time-shift was happening and the Big Birth of a new universe ( or galaxy) was taking place. Besides by-passing singularity, it is clear that Big Birth Theory does not possess "' the black hole information loss paradox"' [11].

When we go on $\mathrm{x}$-axis towards the left side below the level ' $g$ '", $t_{\lambda}$ decreases to minimum levels while $t_{f}$ reaches maximum levels meaning time flows at an extremely high velocity.If we were able to observe a particle there, we would see everything happening at an infinite pace. We would think ourselves as watching the future of that particle since the time velocity of the particle is higher than ours. To understand the situation better consider a wheel of fortune on which a small object placed. In this case the wheel represents the time, while the object placed on it represents our particle. When the wheel turns around at very high speeds $\left(t_{f}\right.$ approaches maximum) we as observers from outside can not determine the objects position at a given time exactly. We can not even see it at very high speeds. Hence at a certain moment of the time we are not certain where the object is. There is a way to see where the object is; we place ourselves on a similar wheel and increase our speed till we are sure for the place of the object. But this time the objects speed becomes vague since the speed difference between us disappears. This means that it is not possible to measure both the velocity and position of a particle simultaneously. It seems that the condition which is known as the Heisenberg Uncertainity Principle [12] is the natural result of the difference between the time wavelengths of ours and that of particles at quantum scale.

Considering the object on the wheel of fortune again, it can be present at anywhere on the wheel until we observe it. So, we -from outside- are looking to the future of the object in a sense and until we observe it the object can be present at anywhere on the wheel at a given time moment with a certain probability and the sum of the probabilities of being at all possible locations is always 1 for the object. This is in accordance with wave function and unitarity.

For the interpretation of the situation in double-slit experiment we can say that when there is no observer, the situation is a future event ( $t_{f}$ of the particle is greater than that of ours) and the probabilities that the electron or photon passes through each slit is equal and the reality is that the electron or photon behave as a wave and passes through both slits. In contrary when the electron or photon is observed, the event isn't in future anymore, one of the probabilities is realised and wave function collapses and electron or photon behave as a particle now.

How can we explain quantum entanglement? When two particles are in close proximity or interact with eachother, actually they equalise their time wavelength and time frequency. So knowing momentum, position, or polarisation of one particle of this pair actually means that we know that property for the other wherever or how far it is since they will behave synchronously. 
Since the new time concept presented in this paper describes the time as an external parameter, this new approach conflicts with the background independence of GR and rather treats GR as background dependent. Furthermore, since the time plays of a pivotal role in both GR and QP , and it is impossible to describe a universe without time according to the presented approach, any "timeless" physical approach is rejected by definition.

The time-dependent Schrödinger Equation is reversible, meaning that we can find solutions for propagating either forward or backward in time. But time-symmetric equivalent of this equation corresponds to the same time frequency $\left(t_{f}\right)$ values of its equivalent but has no physical meaning (Fig.3). Making the time values negative does not change the positivity of the time frequency $\left(t_{f}\right)$ values and physically meaningless. Thus, the arrow of time can not be changed (Fig. 4).

In an above section we described the gravity as being the resistance of a mass to move against time. From this perspective, the time itself with its two dimensions can be regarded as a counteracting force against gravity or as a force of repulsive gravity. Thus, the two dimensional time fabric of the universe acts as the source of a form of energy ( dark energy ) for its expansion. We stated above that $t_{\lambda} \times t_{f}$ is constant throughout the universe. It now appears that $t_{\lambda} \times t_{f}$ is the Universal Constant $(\boldsymbol{\Lambda})$ and is equal to $\Lambda=2.036 \times 10^{-35} s^{-2}$ [13].

A prediction of the presented theory here is that the universe will expand at various rates at different regions due to the fact that particular surroundings will create different gravities and cause a different gravity- time wavelength effect yielding various time delays for calculating this rate of expansion ( Hubble Constant) [14]. Regions with high matter density will have higher gravitational effect on time and will have lower expansion rates. Thus even the same measuring method will yield different Hubble Constant values when applied to the gravitationally different parts of the universe where these parts will be different timewise as well.

\section{CONCLUSION}

The present author tried to outline a theory to solve the well known problems of time and quantum gravity. The essence of the theory is that it tackles the time as a two dimensional entity that weaves the fabric of the universe and approaches it as a kind of energy. The theory has inferences derived from the intrinsic characteristics of matter, time and space equilibrium that we probably did not take into account until now. With this perspective it is possible to find the long-sought answers to some of the famous problems of physics.

\section{ACKNOWLEDGMENTS}

I am particularly grateful to Ferhat Dindaroglu for helpful discussions and comments.

\section{REFERENCES}

1. Einstein A .On the Electrodynamics of moving bodies, Annalen der Physik.17 (10): 891-921, (1905).

2. Giese A. Ongoing problems with special and general relativity, and solutions. J. Phys.: Conf. Ser. $1251012017,(2019)$

3. Gott J R. Time Travel in Einstein's Universe: The Physical Possibilities of Travel Through Time.New York: Houghton Mifflin Company. 44 p. (2002)

4. Friedman Y. Extending the Relativity of Time. J. Phys.: Conf. Ser. 437012017 (2013).

5. Okun LB. Mass versus relativistic and rest masses. Am J Phys. 77(5):430-431 (2009).

6. Schewe PF. Newton's second law of motion. Physics Today.60, 6, 28 (2007).

7. Einstein, Albert (1879-1955), autograph letter signed ('Albert') to Michele Besso, [Princeton], 29 July (1953)

8. Mrowczynski, S. Quark-gluon plasma. Acta Physica Polonica B.29 (12); 3711-24.(1998).

9. Cerenkov PA. Visible emission of clean liquids by action of $\gamma$ radiation. Compt Rend Dokl Akad Mauk SSSR. 8: 451 (1934). 
10. Hawking SW. Black hole explosions? Nature. 248,30-31 (1974).

11. Gurevich I. A Simple Explanation of the Information Paradox by the Information Model of a Black Hole. Open Journal of Microphysics 04(03):21-25 (2014).

12. Peckham GD. Illustrating the Heisenberg uncertainty principle. J. Chem. Educ.61, 10, 868, (1984).

13. Carmeli M, Kuzmenko T. Value of the cosmological constant: Theory versus experiment. AIP Conference Proceedings 586, 316, (2001).

14. Bahcall NA. Hubble's Law and the expanding universe. PNAS.112 (11) 3173-3175 (2015). 